TRABAJOS ORIGINALES

\title{
Dinámica, biomasa aérea y composición florística en parcelas permanentes Reserva Nacional Tambopata, Madre de Dios, Perú
}

\author{
Dynamics, aboveground biomass and composition on permanent plots, Tambopata National \\ Reserve. Madre de Dios, Peru
}

\author{
Nadir C. Pallqui ${ }^{1 *}$, Abel Monteagudo ${ }^{1,2}$, Oliver L.Phillips ${ }^{3}$, Gabriela Lopez-Gonzalez ${ }^{3}$, Luciano Cruz ${ }^{1}$, \\ Washington Galiano ${ }^{1}$, Wilfredo Chavez ${ }^{4}$, Rodolfo Vasquez ${ }^{2}$,
}

1 Universidad Nacional de San Antonio Abad del Cusco, Perú.

2 Jardín Botánico de Missouri, Oxapampa, Pasco, Perú.

3 School of Geography, University of Leeds, LS2 9JT, UK.

4 Instituto de Manejo de Agua y Medio Ambiente, Cusco. Perú.

*Autor para correspondencia:

Email Nadir Pallqui: nadircarolina@hotmail.com Email Abel Monteagudo: monteagudomendoza@yahoo.com

Email Oliver L.Phillips: O.Phillips@leeds.ac.uk Email Gabriela Lopez: g.lopez-gonzalez@leeds.ac.uk Email Luciano Cruz M: lucianojul@yahoo.com Email Washington Galiano: wgalianos2000@yahoo.es

Email Wilfredo Chavez H: wilycha@hotmail.com Email Rodolfo Vasquez: neotaxon@yahoo.com

Citación:

Pallqui N., A. Monteagudo, O.L.Phillips, G. LopezGonzalez, L. Cruz, W. Galiano, W. Chavez, R. Vasquez. 2014. Dinámica, biomasa aérea y composición florística en parcelas permanentes Reserva Nacional Tambopata, Madre de Dios, Perú. Revista peruana de biología 21(3): 235 - 242 (Diciembre 2014). doi: http://dx.doi.org/10.15381/ rpb.v21i3.10897

Fuentes de financiamiento:

El presente trabajo fue financiado por:

Beca de la Universidad Nacional de San Antonio Abad del Cusco, Facultad de Ciencias Biológicas otorgada a Nadir C. Pallqui.

Proyecto RAINFOR (Red Amazónica de Inventarios Forestales) financiado por la Fundación Moore y a NERC (UK).

Oliver Phillips tuvo una beca avanzada de la ERC y es titular del Premio al Mérito Royal Society-Wolfson.

Informacion sobre los autores:

NCP obtuvo los datos de campo, realizó los análisis y la interpretación de datos y se encargó de la redacción del artículo. AM diseñó el estudio, obtuvo los datos de campo, realizó los análisis y la interpretación de datos. OP lideró el proyecto del cual se tomó parte de la información para este estudio contribuyó en la interpretación de datos, realizó una revisión crítica del contenido. GLG contribuyó con los datos a través de Forestplots, organizó e interpretó los datos y en la redacción del artículo. WC contribuyó con los análisis estadísticos y la interpretación de los datos. WG y RV realizaron una revisión crítica del contenido intelectual.

\section{Resumen}

En este estudio se evaluaron los cambios en la biomasa aérea almacenada y su dinámica en el tiempo en 9 parcelas permanentes pertenecientes a la red de parcelas de RAINFOR (Red Amazónica de Inventarios Forestales) ubicadas dentro del bosque húmedo tropical de la Reserva Nacional Tambopata en la llanura Amazónica. Se tomaron datos en campo con la metodología estandarizada RAINFOR. La biomasa se estimó usando la ecuación para bosques húmedos tropicales de Chave et al. (2005). La dinámica de la biomasa se analizó separándolos por periodos inter censales a partir del año 2003 hasta el 2011. Se registraron 64 familias, 219 géneros y 531 especies. La composición florística es muy similar en todas las parcelas excepto en la parcela de pantano. Son evidentes dos grupos ligeramente distintos y aparentemente relacionados a la edad de las antiguas terrazas fluviales de la zona. La tasa de mortalidad y reclutamiento de individuos presentan valores promedio de $2.12 \pm 0.52 \%$ y $1.92 \pm 0.49 \%$, respectivamente. La tasa de reemplazo es $2.02 \%$ anual. La biomasa aérea almacenada fue de $296.2 \pm 33.9 \mathrm{t} \mathrm{ha}^{-1}$ en promedio. La dinámica de la biomasa muestra una ganancia neta total de $1.96,1.69 \mathrm{y}-1.23 \mathrm{t} \mathrm{ha}^{-1}$ para cada periodo respectivamente. Antes de la sequía de 2010 se encontró un cambio en la biomasa de $1.88 \mathrm{t} \mathrm{ha}^{-1}$ año-1 y post sequía fue -0.18 t ha $^{-1}$ año ${ }^{-1}$ en promedio, aunque la diferencia no fue significativa. El análisis demográfico sugiere un equilibrio dinámico en las parcelas. El balance negativo de biomasa observado para el periodo 2008 - 2011 puede deberse a la sequía del 2010, donde la mitad de las parcelas monitoreadas tuvieron una disminución en las ganancias netas de la biomasa por mortalidad de individuos afectando la composición florística selectivamente.

Palabras clave: RAINFOR; mortalidad; reclutamiento; biomasa; llanura Amazónica; Tambopata.

\section{Abstract}

In this study we evaluated the floristic composition and changes in stored biomass and dynamics over time in 9 permanent plots monitored by RAINFOR (Amazon Forest Inventory Network) and located in the lowland Amazon rainforest of the Tambopata National Reserve. Data were acquired in the field using the standardized methodology of RAINFOR. The biomass was estimated using the equation for tropical moist forests of Chave et al. (2005). Biomass dynamics were analyzed, in three separated periods from 2003 to 2011. 64 families, 219 genera and 531 species were recorded. The tree floristic composition is very similar in all plots except for one swamp plot, although but it is also evident that two slightly different forest communities exist in the rest of landscape, apparently related to the age of the ancient river terraces in the area. Mortality and recruitment of individuals averaged $2.12 \pm 0.52 \%$ and 1.92 $\pm 0.49 \%$, respectively. The turnover rate is $2.02 \%$ per year. Aboveground biomass stored in these forests averages $296.2 \pm 33.9 \mathrm{t} \mathrm{ha}^{-1}$. The biomass dynamics show a total net gain of $1.96,1.69$ and $-1.23 \mathrm{t} \mathrm{ha}^{-1}$ for period respectively. Prior to the drought of 2010 a change in biomass was found $1.88 \mathrm{tha}^{-1} \mathrm{yr}^{-1}$ and post drought was $-0.18 \mathrm{t} \mathrm{ha}^{-1} \mathrm{yr}^{-1}$ on average, though the difference is not significant. Demographic analysis suggests a dynamic equilibrium in the plots. The negative balance of biomass observed for the period $2008-2011$ may be due to the drought of 2010, in which half of the monitored plots experienced negative net biomass change due to mortality of individuals selectively affecting the floristic composition.

Keywords: RAINFOR; lowland Amazon rainforest; mortality; recruitment; aboveground biomass; Tambopata.

$\begin{array}{ll}\text { Presentado: } & \text { 23/07/2014 } \\ \text { Aceptado: } & \text { 05/09/2011 } \\ \text { Publicado online: } & 30 / 12 / 2014\end{array}$

Journal home page: http://revistasinvestigacion.unmsm.edu.pe/index.php/rpb/index

(c) Los autores. Este artículo es publicado por la Revista Peruana de Biología de la Facultad de Ciencias Biológicas, Universidad Nacional Mayor de San Marcos. Este es un artículo de acceso abierto, distribuido bajo los términos de la Licencia Creative Commons Atribución-NoComercial-Compartirlgual 4.0 Internacional.(http://creativecommons.org/licenses/by-nc-sa/4.0/), que permite el uso no comercial, distribución y reproducción en cualquier medio, siempre que la obra original sea debidamente citadas. Para uso comercial, por favor póngase en contacto con editor.revperubiol@gmail.com. 


\section{Introducción}

Los bosques tropicales constituyen alrededor del 7\% de la superficie terrestre, participan de forma importante en el balance de carbono global, contribuyen a regular la concentración de dióxido de carbono en la atmósfera, debido a la gran cantidad de carbono que almacenan (aprox. 55\% de las reservas actuales de carbono en los ecosistemas boscosos del mundo, Pan et al. 2011) y a los grandes flujos de carbono que estos bosques procesan cada ańo, fijan aprox. $33 \%$ de la productividad primaria neta terrestre global (del Grosso et al. 2008).

El estudio de la dinámica de la biomasa y el carbono es fundamental para comprender el papel que éstos bosques desempeñan en el ciclo global de este elemento (Clark et al. 2001). Los valores de biomasa no son similares en cualquier parte de la Amazonia, sino que varían sustancialmente de acuerdo a las condiciones ambientales y físicas de cada zona (Honorio \& Baker 2010). Se reporta menos biomasa aérea viva en el oeste de la Amazonia que se correlaciona con menores cantidades de necromasa, menores densidades de madera, dinámica de bosque más elevada (Baker et al. 2004a, Chao et al.2009, Malhi et al. 2006), mayor fertilidad de suelo y una mala estructura física de suelo (Quesada et al. 2009), estas condiciones se relaciones sustancialmente en la captura y almacenamiento de carbono.

En el presente trabajo se analiza la composición florística y se estima la biomasa aérea en 9 parcelas permanentes pre establecidas dentro de la Reserva Nacional Tambopata para el periodo de 2003 al 2011. Los objetivos de este análisis fueron: a) evaluar la variación florística de los árboles en el paisaje b) evaluar la variabilidad de la biomasa en el espacio y en el tiempo y c) evaluar la sensibilidad o resistencia al evento de sequía que se suscitó el año 2010. Estos resultados permitirían entender cómo los bosques tropicales, principalmente los amazónicos responderían a los cambios globales del clima en el futuro, y buscar óptimas formas de adecuación a estos cambios.

\section{Material y métodos}

Área de Estudio.- El presente estudio se realizó en 9 parcelas permanentes establecidas por RAINFOR en las inmediaciones del Albergue Explorer's Inn, en la margen derecha del río Tambopata cerca de la desembocadura del río La Torre dentro de la Reserva Nacional Tambopata, en la provincia de Tambopata del departamento de Madre de Dios, Perú (Fig. 1). Según el sistema de Zonas de Vida de Holdridge las parcelas estan ubicadas en un Bosque Húmedo Tropical entre los 197 y 225 m de altitud, la temperatura media anual varía entre 25 y $26.5^{\circ} \mathrm{C}$, los meses de estiaje entre junio y setiembre y el promedio de precipitación anual es mayor a 2000 milímetros (SERNANP 2012). Los sitios de estudio incluyen bosques inundables (pantano) ó sitios bajos, "bajíos" y bosques que nunca se inundan o "Tierra Firme", los orígenes de los suelos son del Pleistoceno o Holoceno (Phillips et al. 2003) (Tabla1)

Métodos de campo.- En cada censo se midieron todos los árboles con diámetro $\geq 10 \mathrm{~cm}$ POM (usualmente $1.30 \mathrm{~m}$ sobre el nivel del suelo), siguiendo la metodología estandarizada para la remedición de parcelas permanentes de RAINFOR (Phillips \& Baker 2002). Los datos de estas parcelas se almacenan y manejan en la base de datos ForestPlots.net (Lopez-Gonzalez et al. 2011).

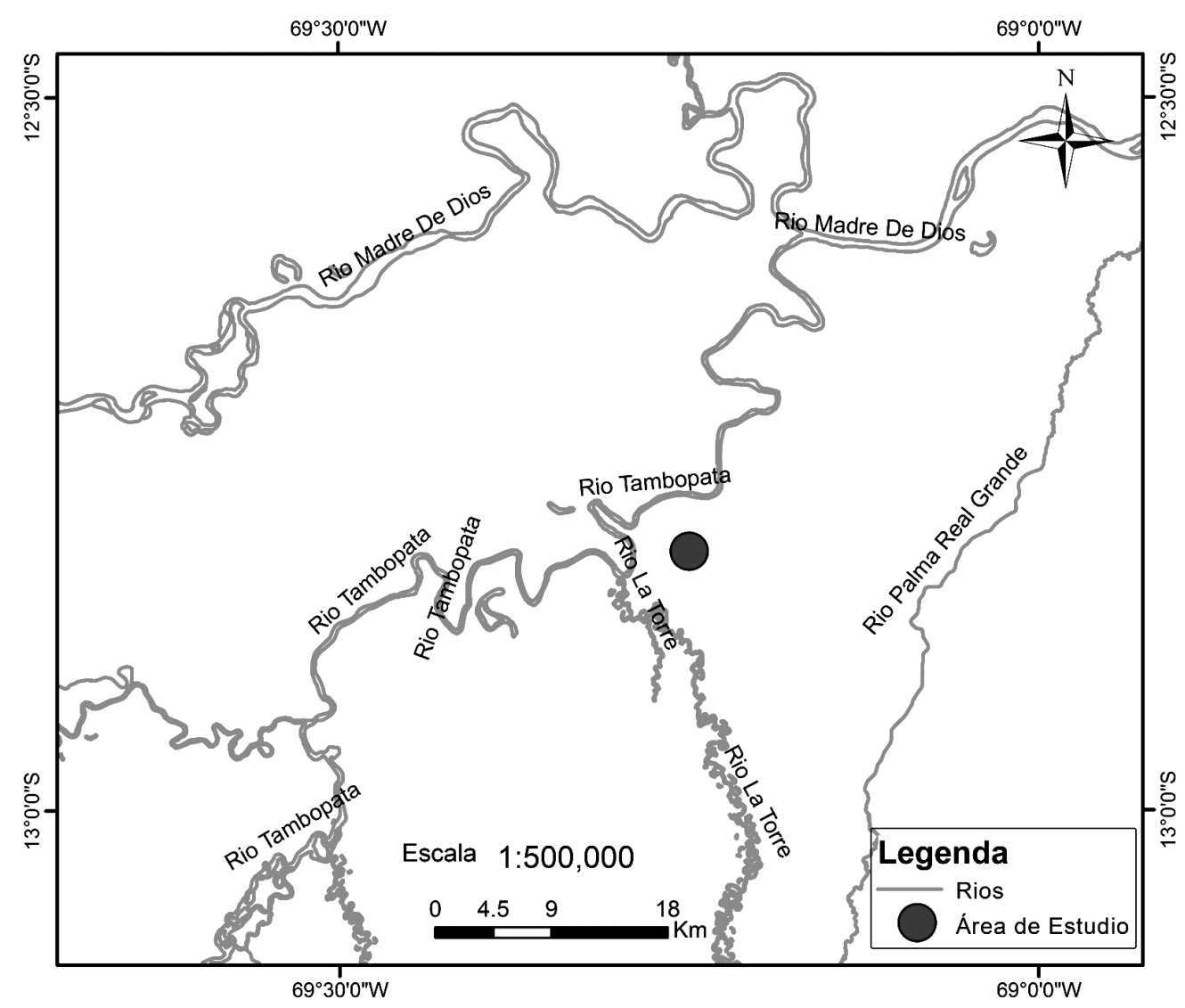

Figura 1: Ubicación de las parcelas permanentes en la Reserva Nacional Tambopata. 
Tabla 1. Características de las parcelas y clasificación y tipo de suelos den cada una de las parcelas y años de medición considerados en la Reserva Nacional Tambopata.

\begin{tabular}{ccccccc}
\hline Parcela & Tamaño & Latitud & Longitud & Altitud $(\mathbf{m})$ & Tipo de Suelo & $\begin{array}{c}\text { Clasificación de } \\
\text { Suelos }\end{array}$ \\
\hline TAM-01 & 1 ha & $12^{\circ} 50^{\prime} 64^{\prime \prime}$ & $69^{\circ} 17^{\prime} 28^{\prime \prime}$ & 205 & Terraza reciente & Alisol \\
TAM-02 & 1 ha & $12^{\circ} 50^{\prime} 10^{\prime \prime}$ & $69^{\circ} 17^{\prime} 16^{\prime \prime}$ & 210 & Tierra firme en Terraza Aluvial Vieja & Alisol \\
TAM-03 & 0.58 ha & $12^{\circ} 50^{\prime} 22^{\prime \prime}$ & $69^{\circ} 16^{\prime} 68^{\prime \prime}$ & 205 & Terraza reciente en Pantano y Borde de Pantano Arcilloso & Gleysol \\
TAM-04 & 0.42 ha & $12^{\circ} 50^{\prime} 22^{\prime \prime}$ & $69^{\circ} 16^{\prime} 68^{\prime \prime}$ & 210 & Terraza reciente & Gleysol \\
TAM-05 & 1 ha & $12^{\circ} 49^{\prime} 81^{\prime \prime}$ & $69^{\circ} 16^{\prime} 23^{\prime \prime}$ & 220 & Tierra firme Arenoso arcilloso & Cambisol \\
TAM-06 & 1 ha & $12^{\circ} 50^{\prime} 31^{\prime \prime}$ & $69^{\circ} 17^{\prime} 76^{\prime \prime}$ & 200 & Terraza reciente & Alisol \\
TAM-07 & 1 ha & $12^{\circ} 49^{\prime} 54^{\prime \prime}$ & $69^{\circ} 15^{\prime} 66^{\prime \prime}$ & 225 & Tierra firme Arenoso Arcilloso & Cambisol \\
TAM-08 & 1 ha & $12^{\circ} 49^{\prime} 57^{\prime \prime}$ & $69^{\circ} 16^{\prime} 16^{\prime \prime}$ & 220 & Tierra firme Arenoso Arcilloso & Cambisol \\
TAM-09 & 1 ha & $12^{\circ} 49^{\prime} 86^{\prime \prime}$ & $69^{\circ} 17^{\prime} 10^{\prime \prime}$ & 197 & Tierra firme Arenoso Arcilloso & Cambisol \\
\hline
\end{tabular}

Análisis de datos.- Los datos fueron compilados en una matriz organizada por especies y número de individuos para cada parcela extraídos de la base de datos: http://www.forestplots. net (Lopez-Gonzalez et al. 2011, 2012). Los nombres científicos fueron revisados y actualizados con claves, listados y TROPICOS (http://www.tropicos.org/). Los géneros y familias fueron basados en la clasificación filogenética de las Angiospermas, APG III (2009).

Basados en la composición y abundancia de las especies en cada parcela de los inventarios, se aplicó un análisis de conglomerados de tipo jerárquico mediante la distancia euclideana y agrupamiento Ward.

Las tasas de mortalidad y reclutamiento anuales se estimaron por separado utilizando procedimientos estándar que utilizan los modelos logarítmicos que asumen una probabilidad constante de la mortalidad y el reclutamiento a través de cada período de inventario (Phillips \& Gentry 1994) mediante los siguientes modelos matemáticos:

$$
\begin{aligned}
& \mathrm{TR}=[\ln (\mathrm{No}-\mathrm{Nm}+\mathrm{Nr})-\ln (\mathrm{No}-\mathrm{Nm})] / \Delta \mathrm{t}(1) \\
& \mathrm{TM}=[\ln (\mathrm{No})-\ln (\mathrm{No}-\mathrm{Nm})] / \Delta \mathrm{t}(2)
\end{aligned}
$$

Donde $T R$ es la tasa de reclutamiento, $T M$ es la tasa de mortalidad, $\Delta t$ es la diferencia entre dos periodos de medición, $N m$ es el número de Individuos muertos en una determinada área, No es el número de individuos al comienzo del periodo y $\mathrm{Nr}$ es el número de individuos reclutados en el segundo periodo.

Las tasas de reemplazo de cada período fueron representadas por la media de reclutamiento y mortalidad. (Phillips et al. 1994).

$\mathrm{TRE}=\overline{\mathrm{X}}$ (tasa de mortalidad, tasa de reclutamiento) (3)

La biomasa aérea $\left(A G B_{e s t}\right)$ se estimó para cada árbol en función al: diámetro $(D)$ y a la gravedad específica de madera $(\boldsymbol{\rho})$ siguiendo un modelo alométrico para árboles tropicales (Chave et al. 2005):

$$
\begin{aligned}
& <\mathrm{AGB}>_{\text {est }}=\rho\left[\operatorname { e x p } \left(-1.499+2.148 \ln (\mathrm{D})+0.207(\ln (\mathrm{D}))^{2}\right.\right. \\
& \left.-0.0281(\ln (\mathrm{D}))^{3}\right)(4)
\end{aligned}
$$

La densidad $\rho$ para cada árbol es estimada utilizando los datos reportados para el Neotrópico en la Global Wood Density Database donde no hay datos a nivel de especie se usó una media global a nivel de especie $\left(0.62 \mathrm{~g} / \mathrm{cm}^{3}\right)$ (Chave et al. 2009; Zanne et al. 2009).
Para comparar las medias de biomasa entre los años de evaluación se usó el análisis de varianza (ANOVA) de un factor. La comparación de la variación de la biomasa entre los periodos de evaluación antes y después de un periodo de sequía, se realizó mediante una prueba no paramétrica de Wilcoxon para muestras pareadas, con un nivel de confianza de 0.05\%. Los análisis se realizaron utilizando el software InfoStat 2013 (Di Renzo et al. 2013)

\section{Resultados}

Composición florística.- Se registraron 64 familias, 219 géneros y 531 especies. En promedio 582 individuos y 157 especies pueden encontrarse en cada parcela de 1 ha.

La Figura 2 resulta del análisis de composición florística que fija un criterio de corte arbitrario en la distancia de 195.3 (que representa el 50\% de la máxima distancia encontrada), la parcela TAM-03 (Grupo 1) se separa del resto, conformando un grupo independiente donde predomina las especies Lueheopsis hoehnei y Mauritia flexuosa. Las parcelas TAM-01, TAM-02 y TAM-06 conforman un grupo homogéneo (Grupo 2); un grupo mayor se conforma por las parcelas TAM-04, TAM-05, TAM-07, TAM08 y TAM-09 (Grupo 3), donde se puede observar dos sub grupos más homogéneos conformados por TAM-07, TAM-05 y TAM-09, TAM-08 y TAM-04.

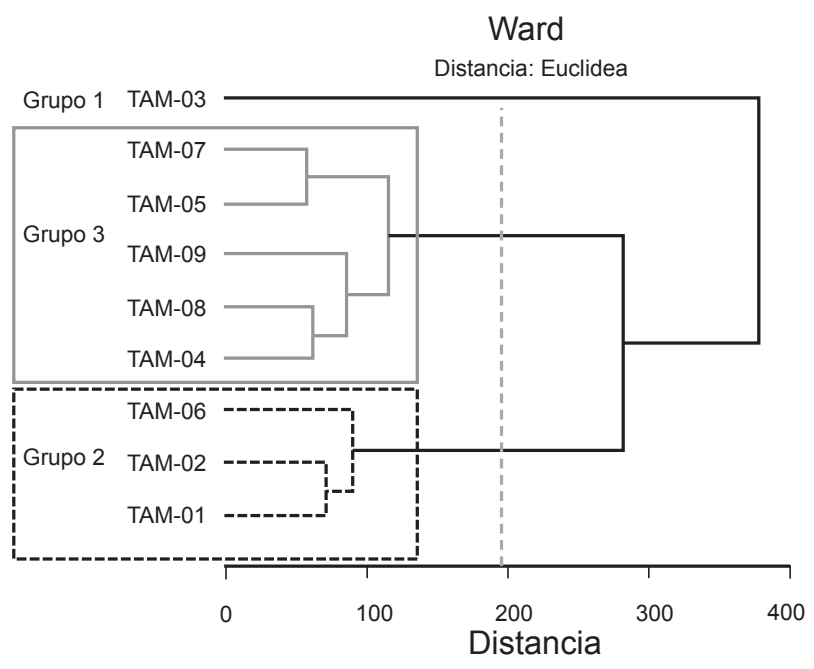

Figura 2: Análisis de agrupamiento de la composición florística por parcela. Se evidencia la formación de tres grupos principales de acuerdo a la composición florística de las parcelas en la Reserva Nacional Tambopata. 
Tabla 2. Dinámica de Individuos del 2003 al 2011: densidad arbórea, tiempo de medición, tasas de mortalidad, reclutamiento y recambio para cada parcela dentro de la Reserva Nacional Tambopata. *TAM-09 fue instalada en el año 2010

\begin{tabular}{|c|c|c|c|c|c|c|c|c|}
\hline Parcela & 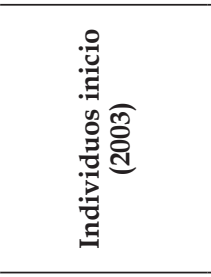 & 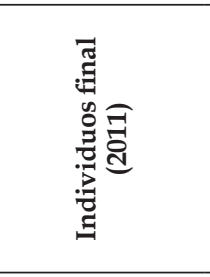 & 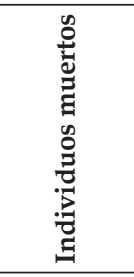 & 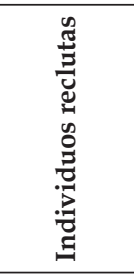 & 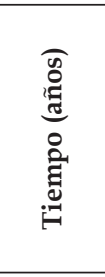 & 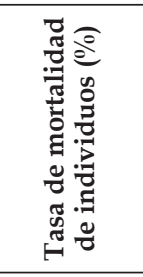 & 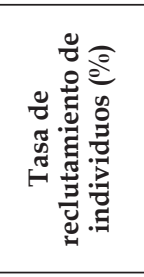 & 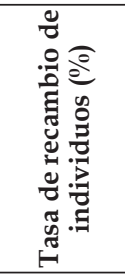 \\
\hline TAM-01 & 600 & 595 & 85 & 81 & 8 & 1.91 & 1.83 & 1.87 \\
\hline TAM-02 & 654 & 658 & 115 & 118 & 8 & 2.42 & 2.47 & 2.45 \\
\hline TAM-03 & 356 & 357 & 37 & 39 & 8 & 1.37 & 1.44 & 1.41 \\
\hline TAM-04 & 301 & 286 & 47 & 30 & 8 & 2.12 & 1.40 & 1.76 \\
\hline TAM-05 & 531 & 526 & 78 & 72 & 8 & 1.99 & 1.84 & 1.91 \\
\hline TAM-06 & 629 & 659 & 74 & 103 & 8 & 1.56 & 2.13 & 1.85 \\
\hline TAM-07 & 524 & 507 & 115 & 97 & 8 & 3.10 & 2.66 & 2.88 \\
\hline TAM-08 & 527 & 512 & 98 & 83 & 8 & 2.57 & 2.21 & 2.39 \\
\hline TAM-09* & 556 & 552 & 11 & 7 & 1 & 2.00 & 1.28 & 1.64 \\
\hline TOTAL & 4122 & 4652 & 660 & 630 & 65 & 19.04 & 17.26 & 18.15 \\
\hline Promedio \pm D.E. & $515.25 \pm 125.98$ & $516.89 \pm 125.76$ & $73 \pm 35.60$ & $70 \pm 36.99$ & $7 \pm 2.33$ & $2.12 \pm 0.52$ & $1.92 \pm 0.49$ & $2.02 \pm 0.46$ \\
\hline
\end{tabular}

Tasa de mortalidad anual de individuos.- Las parcelas presentan un rango entre $1.37-3.10 \%$, siendo la parcela TAM07 la que tiene la mayor tasa de mortalidad. Se registraron 660 individuos muertos durante 8 años en las parcelas de la Reserva Nacional Tambopata, donde el promedio para las 9 parcelas evaluadas es $2.12 \pm 0.52 \%$ (Tabla 2 ).

Tasa de reclutamiento anual de individuos.- El rango de la tasa de reclutamiento va desde 1.28 a $2.66 \%$ este valor máximo es alcanzado por TAM-07, la tasa de reclutamiento anual promedio es de $1.92 \pm 0.49 \%$ con el registro de 630 individuos reclutas (Tabla 2 ).

Tasa de recambio de individuos.- Resulta del promedio de la tasa de mortalidad y la tasa de reclutamiento. Predeciblemente la parcela TAM-07 es la que tuvo un proceso de cambio de individuos más veloz presentando un valor de $2.88 \%$; el rango de la tasa de recambio varía entre 1.41 y $2.88 \%$ y el valor promedio es de $2.02 \pm 0.46 \%$. Cabe mencionar que la tasa de recambio determina la dinámica del bosque e indirectamente podría condicionar la diversidad de especies que pueda contener un área dada (Tabla 2).

Estimación de la biomasa aérea almacenada.- A lo largo de los ańos de evaluación se registró el diámetro a un total de 6794 individuos $\geq 10 \mathrm{~cm}$ de diámetro. Se estimó la biomasa individual de todos los individuos, posteriormente se totalizo por hectárea obteniendo un promedio total de $296.2 \pm 33.9 \mathrm{t}$ ha $^{-1}$ de biomasa aérea almacenada (Tabla 3 ), valor que es relativamente alto comparado con otros bosques amazónicos. La parcela que almacena mayor cantidad de biomasa a través de los años de medición es TAM-03 con $342.4 \mathrm{t} \mathrm{ha}^{-1}$ año $^{-1}$ y la parcela que aporta con la menor cantidad de biomasa es TAM-08 con $258.1 \mathrm{t} \mathrm{ha}^{-1}$ año $^{-1}$. Las especies arbóreas de grandes diámetros influyen considerablemente en el comportamiento de la biomasa

Tabla 3. Biomasa estimada en el intervalo del 2003 al 2011: Tasa de cambio de la biomasa para cada parcela dentro de la Reserva Nacional Tambopata. *TAM-09 fue instalada en el año 2010.

\begin{tabular}{|c|c|c|c|c|c|c|}
\hline \multicolumn{7}{|c|}{ Intervalo completo (2003-2011) } \\
\hline Parcela & $\begin{array}{l}\text { AGB Inicial } \\
\qquad\left(\mathrm{t} \mathrm{ha}^{-1}\right)\end{array}$ & $\begin{array}{l}\text { AGB Final } \\
\qquad\left(\mathrm{t} \mathrm{ha}^{-1}\right)\end{array}$ & $\begin{array}{l}\text { AGB promedio } \\
\left(\mathbf{t ~ h a}^{-1}\right)\end{array}$ & $\begin{array}{l}\text { Tiempo } \\
\text { (años) }\end{array}$ & $\begin{array}{l}\text { AGB cambio } \\
\left(\mathrm{t} \mathrm{ha}^{-1}\right)\end{array}$ & $\begin{array}{l}\text { AGB cambio, por } \\
\text { año }\left(\mathrm{t} \mathrm{ha}^{-1}\right)\end{array}$ \\
\hline TAM-01 & 253.3 & 273.7 & 263.5 & 8 & 20.3 & 2.5 \\
\hline TAM-02 & 284.9 & 257.5 & 271.2 & 8 & -27.4 & -3.4 \\
\hline TAM-03 & 321.3 & 363.4 & 342.4 & 8 & 42.1 & 5.3 \\
\hline TAM-04 & 336.8 & 347.3 & 342.0 & 8 & 10.5 & 1.3 \\
\hline TAM-05 & 283.1 & 311.1 & 297.1 & 8 & 28.0 & 3.5 \\
\hline TAM-06 & 308.7 & 352.1 & 330.4 & 8 & 43.4 & 5.4 \\
\hline TAM-07 & 315.6 & 263.1 & 289.4 & 8 & -52.5 & -6.6 \\
\hline TAM-08 & 254.7 & 261.4 & 258.1 & 8 & 6.7 & 0.8 \\
\hline TAM-09* & 276.3 & 266.7 & 271.5 & 1 & -9.6 & -9.6 \\
\hline \multicolumn{3}{|c|}{ Promedios \pm D.E. } & $296.2 \pm 33.9$ & $7.2 \pm 2.33$ & $6.8 \pm 32$ & $-0.08 \pm 5.3$ \\
\hline \multicolumn{3}{|c|}{ Intervalo de confianza, 95\% } & 22.2 & 1.52 & 20.9 & 3.5 \\
\hline
\end{tabular}




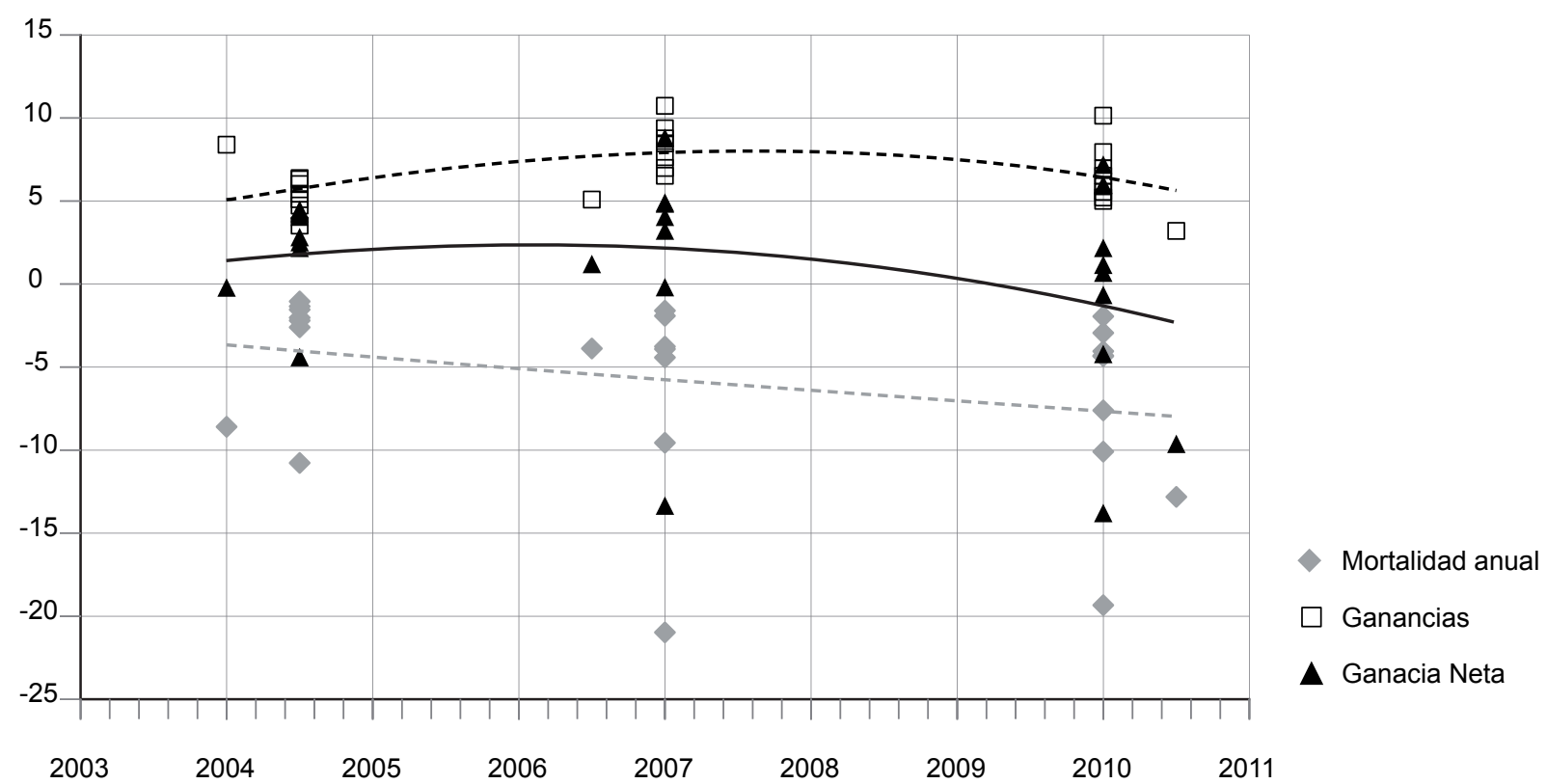

Figura 3. Tendencia de ganancias, mortalidad y balance netos de la biomasa aérea en las parcelas permanentes de la Reserva Nacional Tambopata. La línea negra punteada determina el crecimiento de la biomasa por reclutamiento (cifras positivas), en gris punteada la pérdida de biomasa por mortalidad (cifras negativas) y en linea negra entera, el balance o ganancia neta entre los dos procesos. Cada punto representa el comportamiento de cada parcela dentro de un intervalo. Las líneas representan el promedio (promedios polinomios)

en tiempo y espacio. El promedio de la biomasa aérea (AGB) de árboles por año de medición toma valores crecientes partiendo con 294.8 t ha $^{-1}$ en el 2003 alcanza su mayor valor el ańo 2008 con $304.3 \mathrm{t} \mathrm{ha}^{-1}$ luego decrece para el 2011 con $299.6 \mathrm{t} \mathrm{ha}^{-1}$, sin embargo no presentan diferencias estadísticamente significativas $(\mathrm{F}=0.113)$ en sus medias en un nivel de confianza del $95 \%$.

Dinámica de la biomasa aérea almacenada.- Se evaluó la dinámica de la biomasa en tres periodos inter censales sobre su: pérdida de biomasa por mortalidad, ganancia por reclutamiento y la ganancia neta (ganancia menos la pérdida) donde durante los dos primeros periodos intercensales 2003 - 2006 y 2006 - 2008 las parcelas de Tambopata ganaron biomasa en valores promedio de 1.96 y $1.69 \mathrm{t} \mathrm{ha}^{-1}$ año $^{-1}$, mientras que para el último periodo 2008 - 2011 la ganancia neta fue negativa de $-1.23 \mathrm{t} \mathrm{ha}^{-1} \mathrm{año}^{-1}$. La Figura 3 evidencia de la variabilidad espacial y temporal en la dinámica de la biomasa en las parcelas permanentes de la Reserva Nacional Tambopata, la cual depende a su vez de la dinámica poblacional propia del bosque, aportando con datos esenciales para estudios de almacenes y flujos de carbono en la Amazonía, así como tendencias a largo plazo de los bosques tropicales maduros.

Biomasa aérea pre y post sequía del 2010.- Los análisis pre y post sequía demuestran que previo al 2010 hubo un incremento de biomasa de $1.88 \mathrm{t} \mathrm{ha}^{-1}$ año $^{-1}$ por contraste, en el periodo post sequía hubo una pérdida de biomasa de $-0.18 \mathrm{t}$ $\mathrm{ha}^{-1}$ año $^{-1}$, estas pérdidas se da principalmente por la ocurrencia y aceleración de la tasa de mortalidad arbórea y disminución en la tasa de crecimiento, con una reducción de 13.24 toneladas de biomasa aérea en las 8 ha.

En la Figura 4 se observa cambios en la distribución de los datos, después del 2010 la mediana de la biomasa aérea almacenada de las 8 parcelas evaluadas disminuye y se incrementa la media, producto que entre ambos periodos cambia la dis- tribución de los valores de biomasa, siendo más dispersa en el periodo después 2010.

El valor $p>0.99$ para un nivel de significación $\alpha=0.05$, indica el rechazo de la hipótesis de igualdad de medias por lo que no existen diferencias estadísticamente significativas entre ambos

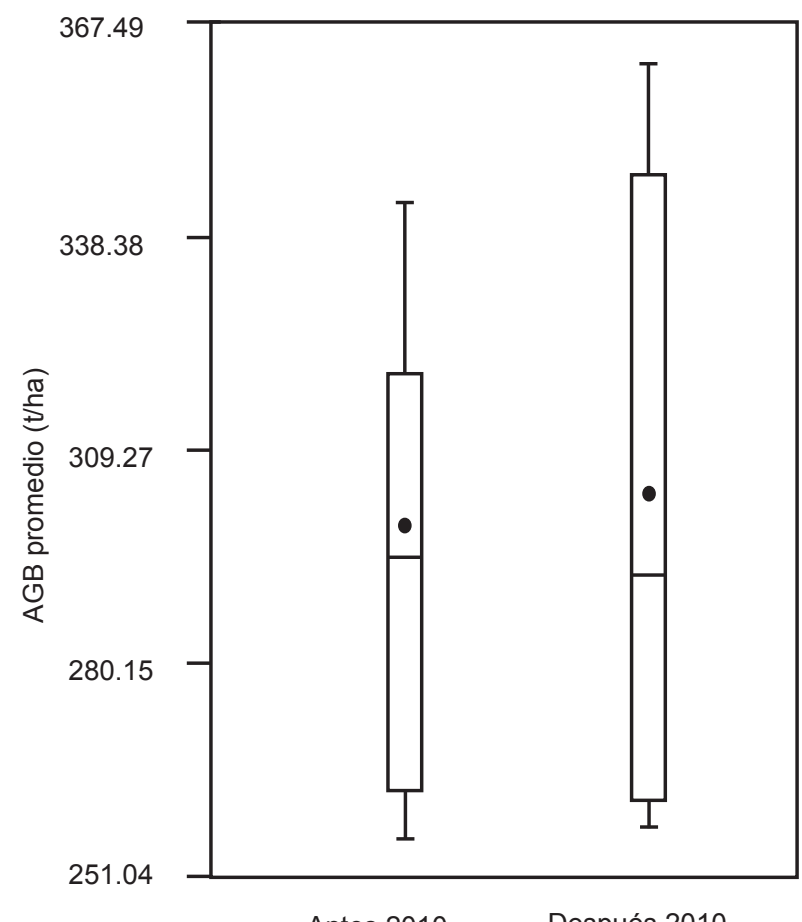

Antes 2010

Después 2010

Figura 4. Tasa de cambio de biomasa aérea antes y después del año 2010. Excepto TAM-09 que fue instalada en el 2010. 
periodos de evaluación de la biomasa promedio. En tal sentido, se puede afirmar que los efectos de los periodos de sequía presentes en el ámbito de la Amazonia evaluada, no repercutieron significativamente en los incrementos de biomasa.

El mecanismo de pérdida de biomasa por mortalidad fue principalmente del tipo "muerto en pie" 42.26\%. En relación a la composición florística las especies: Iriartea deltoidea (Arecaceae), Pourouma minor (Urticaceae) y Roucheria punctata (Linaceae) se identificaron como las más sensibles a la sequía.

\section{Discusión}

Posiblemente la agrupación de parcelas en cuanto a composición florística esté relacionada directamente con la clase de suelo a la que pertenece ya que el grupo (1) son suelos tipo Gleisol, el segundo (2) de tipo Cambisol y finalmente el tercero (3) es de tipo Alisol (Quesada et al. 2009, 2011); incluso estos grupos se relacionan con la antigüedad de las superficies: Pleistoceno (terrazas antiguas) concuerda con el grupo (1) y (2) y Holoceno (llanura muy reciente) representa al grupo (3) de acuerdo a los estudios hechos por Phillips et al. 2003.

Las familias más importantes dentro de las parcelas de Tambopata fueron: Fabaceae, Moraceae, Annonaceae, Lauraceae, Sapotaceae, Myristicaceae, Rubiaceae, Malvaceae y Arecaceae los cuales coincidieron con las familias reportadas por Vásquez y Rojas (2006), Gentry (1989), Terborgh \& Andresen (1998) y ter Steege (2009) en la Amazonía baja peruana. Asimismo en el Pitman et al. (2005) mencionan que más de la mitad de árboles de Madre de Dios pertenecen a estas familias.

Las especies arbóreas que dominaron en las parcelas evaluadas fueron: Iriartea deltoidea, Astrocaryum murumuru, Euterpe precatoria (Arecaceae), Leonia glycycarpa (Violaceae), Pseudolmedia laevis, Pseudolmedia laevigata, Perebea mollis subsp. lecithogalacta (Moraceae), Pourouma minor (Urticaceae), Virola calophylla (Myristicaceae), Aspidosperma parvifolium (Apocynaceae),Siparuna decipiens (Siparunaceae), Apeiba aspera (Malvaceae), Tachigali poeppigiana (Fabaceae), Pouteria torta (Sapotaceae); como las principales, las cuales se incluyen en la lista de las 150 especies arbóreas más comunes de tierra firme en Madre de Dios por Pitman et al. (2001).

TAM-03 ubicada en una zona pantanosa se aísla de las demás parcelas por tener menor riqueza de especies y dominancia de una especie que es Lueheopsis hoehnei, especie de madera dura y crecimiento lento. Gentry (1989) y Nebel et al. (2000) mencionaron que muy pocas especies de la región toleran la inmersión prolongada por un drenaje deficiente condicionado por la poca cantidad de oxigeno presente en el suelo, condiciones extremas que resultan en una mínima diversidad de especies que sus contrapartes no inundadas.

La tasa de reemplazo, los rangos encontrados por Phillips y Gentry (1994) en la misma zona de estudio varía entre 0.82 $-2.61 \%$, posteriormente en el 2004 ofrece un rango de 0.95 $-2.55 \%$, variando a lo encontrado en la presente investigación que presenta un rango entre 1.41 y $2.88 \%$. Así parece que las tasas tuvieron un ligero incremento durante los últimos veinte años, lo que coincide con la conclusión hecha por Phillips y Gentry (1994) y Phillips et. al. (2004) en los que considera que el incremento de la tasa de reemplazo a través del tiempo en los bosques tropicales es dos veces mayor que en la década de los 50 .
Phillips y Gentry (1994) también afirmaron que cuanto más rápido crece un bosque, más rápido morirán sus árboles y más rápida será la tasa de recambio, estas afirmaciones se ajustan al presente estudio donde se halló el valor mínimo de $1.41 \%$ para TAM-03 siendo por tanto la menos dinámica presentando la menor riqueza de especies dominada por Lueheopsis hoehnei situada en un bosque inundable y lo opuesto ocurre en TAM-07 donde las tasas de reclutamiento y mortalidad son más altas y por tanto mayor tasa de recambio de $2.88 \%$.

De acuerdo a los mapas de biomasa la región oeste de la Amazonía según Saatchi et al. (2007), presenta un rango entre 200 a $300 \mathrm{t} \mathrm{ha}^{-1}$, en cambio, para Malhi et al. (2006) y Baker et al. (2004a) la biomasa varía entre 250 y $350 \mathrm{t} \mathrm{ha}^{-1}$ año $^{-1} \mathrm{ca}-$ tegorizándolo como biomasa media, la presente investigación coincide con estos ya que los rangos de biomasa varían entre 258.1 y $342.4 \mathrm{t} \mathrm{ha}^{-1}$ a lo largo del tiempo evaluado. Estos niveles de biomasa coinciden con niveles bajos de necromasa (Araujo-Murakami et al. 2011) y densidad de madera (Baker et al. 2004b).

Los rangos de biomasa aérea para los bosques amazónicos en el Perú oscilan desde los 216.96 y 299.20 t ha $^{-1}$ (Baker et al. 2004a); Lopez-Gonzalez et al. (2012) estimaron un promedio de $252.2 \pm 11.04 \mathrm{t} \mathrm{ha}^{-1}$ para los bosques maduros de la Amazonía peruana y específicamente para los bosques de Tambopata valores entre 216.19 y 270.89 t ha $^{-1}$ siendo diferentes a los valores que hemos encontrado, debido a que en su estimación se utilizan además datos de altura, comprobando que las ecuaciones para estimar la biomasa también son otro factor de variabilidad. Incluso Baker et al. (2004b) y Vilanova (2007) suponen que la ecuación de Chave et al. (2005) que solamente considera diámetro y densidad de la madera podría sobrestimar la biomasa aérea. Ya que los árboles del suroeste de la Amazonia son más bajos en relación a los del este y centro de la Amazonia, surge la incertidumbre por la estimación de la biomasa área, por tanto se necesita generar ecuaciones alométricas regionales que relacionen el diámetro y la altura en base a mediciones directas.

De acuerdo a Phillips et al. (2009), quienes analizaron la sensibilidad a la sequía del 2005, los bosques maduros de la Amazonía peruana antes del 2005 tenían una tasa de cambio promedio de 1.33 t ha- 1 año-1 y después del 2005 fue de 0.26 t ha-1 ańo-1; en la presente investigación respecto de la sequía del 2010 encontramos que la tasa de cambio de biomasa para el periodo pre-sequía se estimó en 1,88 t ha-1 año-1 y para el periodo post sequía en - 0,18 t ha- 1 año- 1 por lo que se presume un efecto negativo a causa de la sequía del 2010 (Lewis et al. 2011). Sin embargo no se puede afirmar dicha hipótesis con tan pocas parcelas de estudio y con un corto periodo de análisis que no permite la identificación de tendencias a largo plazo.

La mortalidad de Iriartea deltoidea se tiene que resaltar ya que esta especie fue la más sensible a los efectos de la sequía del 2010, lo que también se observó en la sequía del 2005 donde murieron miles de estas palmeras en la Amazonía; de acuerdo a Phillips et al. (2009), esta sensibilidad se le puede atribuir a que estas palmeras tienen raíces superficiales que no penetran profundamente y como hay déficit de agua en el mismo suelo no hay como estas capten este recurso, además su forma de muerte fue "en pie" y con el paso del tiempo se doblaron. 


\section{Conclusiones}

Las parcelas de Tambopata tienen una gran representatividad de los bosques de toda la reserva en cuanto a la composición florística de cada tipo de bosque. La conformación de grupos permite establecer agrupaciones de parcelas florísticamente homogéneas, la cual puede estar determinada por las características ambientales presentes en cada una de las parcelas y por tanto también se puede asumir condiciones ambientales homogéneas para cada grupo.

En relación a la dinámica forestal, los bosques de la Amazonia de Madre de Dios son bosques rápidos comparados con otros bosques se localizan en las regiones Este y Centro. Las parcelas incluidas en este estudio han mostrado un incremento en las tasas de reclutamiento y mortandad, las tasas de mortalidad superan a las tasas de reclutamiento. Es probable que los cambios en la composición de especies y función del bosque se presenten a la par de los cambios observados en la estructura y dinámica de los bosques de las parcelas de Tambopata.

La biomasa aérea almacenada dentro de la Reserva Nacional Tambopata es media comparada con otros bosques amazónicos, el 50\% de esta biomasa viene a ser carbono almacenado, por lo cual estos bosques cumplen el importante papel de stocks de carbono. Se sugiere que la composición de los bosques amazónicos es un factor determinante de los patrones anuales de acumulación de biomasa.

A pesar de que las parcelas permanentes en estudio han ganado biomasa en los primeros años de evaluación (siendo sumideros de carbono), este proceso sufrió cambios en el último periodo 2008-2011 el cual presumiblemente se deba a la sequía anómala del 2010 ya que coincide con la disminución de biomasa en la red de parcelas estudiadas debido al aumento de mortalidad y una reducción del crecimiento afectando la composición florística selectivamente de acuerdo a la resiliencia de cada especie, sin embargo estas pérdidas no son significativas.

\section{Agradecimientos}

Los autores expresan su agradecimiento a la Fundación Moore y a NERC (UK), por brindar la subvención económica para este estudio dentro del proyecto RAINFOR (Red Amazónica de Inventarios Forestales). El Profesor Oliver Phillips tiene el apoyo de una beca avanzada de la ERC y es titular del Premio al Mérito Royal Society-Wolfson. A los colegas e investigadores por su apoyo en el campo a lo largo de los años: Víctor Chama, Antonio Pena, Yuri Huillca, Amador Pfuro, Ítalo Treviño, Eurídice Honorio, Geertje van der Heijden, Magnolia Restrepo, Michael García, Néstor Jaramillo, Margaret Stern, Camilo Díaz, Javier Silva, Tim Baker, Ted Feldpausch, Percy Núnez, Alexander Parada, Kate Johnson, Sam Rose, Jim Martin, Ángela Rozas, Virginia Retamozo. Un especial agradecimiento a Forestplots.net por todas las facilidades y acceso otorgadas para la realización de la tesis; al Servicio Nacional de Áreas Naturales Protegidas por el Estado (SERNANP), por las facilidades brindadas al concedernos las respectivas autorizaciones de investigación, al Herbario Selva Central, Oxapampa (HOXA), por el acceso a las colecciones botánicas.

\section{Literatura citada}

APG III. 2009. An update of the Angiosperm Phylogeny Group classification for the orders and families of flowering plants: APG III. Botanical Journal of the Linnean Society 161:105-121. http:/dx.doi.org/10.1111/j.1095-8339.2009.00996.x
Araujo-Murakami A., A.G. Parada, J. Terán, et al. 2011. Necromasa de los bosques de Madre de Dios, Perú; una comparación entre bosques de tierra firme y de bajíos. Revista Peruana de Biología 18(1):113-118. http://dx.doi.org/10.15381/ rpb.v18il.155

Baker T.R., O.L. Phillips, Y. Malhi, et al. 2004a. Increasing biomass in Amazonían forest plots. Philosophical Transactions of the Royal Society of London Series B 359, 353-365. http:/ dx.doi.org/10.1098/rstb.2003.1422.

Baker T.R., O.L. Phillips, Y. Malhi, et al. 2004b. Variation in wood density determines spatial patterns in Amazonían forest biomass. Global Change Biology 10, 545-562. http:/dx.doi. org/10.1111/j.1529-8817.2003.00751.x

Chao K.J., O.L. Phillips, T. Baker, et al. 2009. After trees die: quantities and determinants of necromass across Amazonia. Biogeosciences, 6, 1615-1626, http:/dx.doi.org/10.5194/ bg-6-1615-2009

Chave J., C. Andalo, S. Brown, et al. 2005. Tree allometry and improved estimation of carbon stocks and balance in tropical forests. Oecología 145, 87-99. http:/dx.doi.org/10.1007/ s00442-005-0100-x.

Chave J., D.A. Coomes, S. Jansen, et al. 2009. Towards a worldwide wood economics spectrum. Ecology Letters 124: 351-366. http:/dx.doi.org/10.1111/j.1461-0248.2009.01285.x

Clark D.A., S. Brown, D. W. Kicklighter, et al. 2001. Net Primary Production in Forest: An Evaluation and Sinthesis of Existing Field Data. Ecological Applications, 11, 371-384. http:/ dx.doi.org/10.1890/1051-0761(2001)011[0371:NPPIT $\mathrm{F}] 2.0 . \mathrm{CO} ; 2$

del Grosso, S., W. Parton, T. Stohlgren, et al. 2008. Global potential net primary production predicted from vegetation class, precipitation, and temperature. Ecology 89:2117-2126. http:/dx.doi.org/10.1890/07-0850.1

Di Rienzo J.A., F. Casanoves, M.G. Balzarini, et al. 2013. InfoStat versión 2013. Grupo InfoStat, FCA, Universidad Nacional de Córdoba, Argentina. URL http://www.infostat.com.ar

Gentry A.H. 1989. Checklist of the plants, Zona Reservada de Tambopata, Perú. Reporte Missouri Botanical Garden.

Honorio E. \& T. Baker. 2010. Manual para el Monitoreo del ciclo del Carbono en Bosques Amazónicos. Instituto de Investigaciones de la Amazonía Peruana/ Universidad de Leeds. Lima, 54p. <http://www.rainfor.org/upload/ManualsSpanish/ Honorio_Baker2010\%20Manual\%20carbono.pdf. Acceso 07/05/2013.

Lewis S.L., P.M. Brando, O.L. Phillips, et al. 2011. The 2010 Amazon Drought. Science 331 (6017):554. http:/dx.doi. org/10.1126/science. 1200807.

Lopez-Gonzalez G., S.L. Lewis, M. Burkitt, et al. 2011. ForestPlots. net: a web application and research tool to manage and analyse tropical forest plot data. Journal of Vegetation Science 22 4: 610-613. http:/dx.doi.org/10.1111/j.16541103.2011.01312.x

Lopez-Gonzalez G., S.L. Lewis, M. Burkitt, et al. [2012] en línea. Forest Plots Database. www.forestplots.net. Acceso 01/03/2012.

Malhi Y., D. Wood, T. Baker, et al. 2006. The regional variation of aboveground live biomass in old-growth Amazonían forests. Global Change Biology 12 (7):1107-1138. http:/dx.doi. org/10.1111/j.1365-2486.2006.01120.x

Nebel,G., L. Kvist, J. Vanclay et al. 2000. Dinámica de los bosques de la llanura alluvial inundable de la Amazonía Peruana: Efectos de las perturbaciones e implicancias para su manejo y conservación. Folia Amazónica Vol. 11, 65-97.

Pan Y., R. Birdsey, J. Fang. et al. (2011). A Large and Persistent Carbon Sink in the World's Forests. Sciencen 333, 6045 pp. 988993. http:/dx.doi.org/10.1126/science.1201609.

Phillips O. \& A.H. Gentry. 1994. Increasing turnover through time in tropical forest. Science 263, 954-958. http:/dx.doi. org/10.1126/science.263.5149.954.

Phillips O.L \& T. Baker. 2002. Field manual for plot establishment and remeasurement RAINFOR. Amazon Forest Inventory Network, Sixth Framework Programme 2002-2006.

Phillips O.L., L. Aragão, S. Lewis, et al. 2009. Drought Sensitivity of the Amazon Rainforest. Science 323 5919: 1344-1347. http:/dx.doi.org/10.1126/science.1164033. 
Phillips O.L., T. R. Baker, W. F. Laurance, et al. 2004. Pattern and process in Amazon tree turnover, 1976-2001 in Phil. Trans. R. Soc. Lond. B 359: 381-407pp. http:/dx.doi.org/10.1098/ rstb.2003.1438.

Phillips O.L., P. Nuñez-Vargas, A. Monteagudo, et al. 2003. Habitat association among Amazonian tree species: a landscape-scale approach. Journal of Ecology, 91: 757-775. http:/dx.doi. org/10.1046/j.1365-2745.2003.00815.x

Pitman N., J. Terborgh J., M. Silman, et al. 2001. Dominance and distribution of tree species in upper Amazonian terra firme forests. Ecology 82 8: 2101-2117. http://dx.doi. org/10.1890/0012-9658(2001)082[2101:DADOTS]2.0 .CO;2

Pitman, N., P. Núñez \& J. Terborgh 2005. Árboles Comunes de los Bosques inundados de Madre de Dios. Biodiversidad Amazónica 1 (1): 6-15.

Quesada C. A., J. Lloyd, L.O. Anderson, et al. 2011. Soils of Amazonia with particular reference to the RAINFOR sites. Biogeosciences 8 (6):1415-1440. http:/dx.doi.org/10.5194/ bg-8-1415-2011

Quesada C.A., J. Lloyd, M. Schwarz, et al. 2009. Regional and large-scale patterns in Amazon forest structure and function are mediated by variations in soil physical and chemical properties. Biogeosciences Discuss., 6, 3993-4057. http:/dx.doi.org/10.5194/bgd-6-3993-2009
Saatchi S.S., R.A. Houghton, R.C. Dos Santos Alvala, et al. 2007. Distribution of aboveground live biomass in the Amazon basin. Global Change Biology 13, 816-837. http:/dx.doi. org/ 10.1111/j.1365-2486.2007.01323.x

SERNANP 2012. (en línea) Diagnostico del Proceso de Elaboración del Plan Maestro de la Reserva Nacional Tambopata, Madre de Dios (2011-2016). 128 p. Disponible en: <http://www. sernanp.gob.pe/sernanp/archivos/biblioteca/planes_maestros_2012/PM\%20RNTAMB\%202011-2016.pdf> Acceso $12 / 04 / 12$.

Terborgh J. \& E. Andresen. 1998. The composition of Amazonian forests: patterns at regional and continental scales. Journal of Tropical Ecology 14: 645-664. http://dx.doi.org/10.1017/ S0266467498000455

ter Steege H. 2009. Contribution of Current and Historical Processes to Patterns of Tree Diversity and Composition of the Amazon, in Amazonia: Landscape and Species Evolution: A look into the past (eds C. Hoorn and F. P. Wesselingh), Wiley-Blackwell Publishing Ltd., Oxford, UK. http:/dx.doi. org/10.1002/9781444306408.ch21

Vasquez R. \& R. Rojas, 2006. Plantas de la Amazonía Peruana: Clave para Identificar las Familias de Gymnospermae y Angiospermae. Segunda edición. Arnaldoa. 13(1). 09-258.

Vilanova E. 2007. Impacto de la extracción selectiva de maderas sobre el almacenamiento de carbono en un bosque de la Guayana Venezolana. Revista Forestal Venezolana 51(2)2007.pp.261

Zanne A., G. Lopez-Gonzalez, D.A. Coomes, et al. 2009. Data from: Towards a worldwide wood economics spectrum. Dryad Digital Repository. http:/dx.doi.org/10.5061/dryad.234. 